\title{
Rhizopus Keratitis Associated with Poor Contact Lens Hygiene
}

\author{
David B. Warner $^{\mathrm{a}}$ Hugh E. Wright III ${ }^{\mathrm{a}}$ Eric R. Rosenbaum ${ }^{\mathrm{b}}$ \\ Departments of ${ }^{\mathrm{a}}$ Ophthalmology and ${ }^{\mathrm{b}}$ Microbiology, University of Arkansas for Medical \\ Sciences, Little Rock, Ark., USA
}

\section{Key Words}

Rhizopus keratitis · Contact lens hygiene

\begin{abstract}
We report a case of Rhizopus keratitis in a young woman with poor contact lens hygiene. The mold was highly sensitive to treatment with amphotericin $0.15 \%$ drops, after a relatively prompt diagnosis. Obtaining cultures of both corneal infiltrates and presumably infected contact lenses may help to avoid a delay in proper treatment.

(C) 2016 The Author(s)

Published by S. Karger AG, Basel
\end{abstract}

\section{Case Report}

A 27-year-old white female with a history of poor contact lens hygiene, including continuous wear, presented to our clinic with left eye pain, redness, and worsening vision for 2 days. Visual acuity was initially recorded as counting fingers at 3 feet. Intraocular pressures were within normal limits. Examination revealed severe conjunctival injection with ciliary flush, and a $<1 \times 1 \mathrm{~mm}$ central corneal ulcer with mild stromal thinning. Other findings included a diffuse lymphocytic stromal infiltrate, microcystic edema, and $360^{\circ}$ limbal neovascularization. Scattered small scars were also present indicating previous infection. The anterior chamber was quiescent, and the pupil was round and reactive. Corneal scrapings were sent to the microbiology laboratory for Gram stain and were cultured on blood, chocolate, and potato dextrose agars. Moxifloxacin (Vigamox; Alcon Laboratories, Fort Worth, Tex., USA) $0.5 \%$ drops were initiated hourly. The scrapings grew 5 colonies of a coagulasenegative staphylococcus, which was thought to be a contaminant.

After $48 \mathrm{~h}$ of treatment, the patient developed a $2.5 \times 2.5 \mathrm{~mm}$ feathery, stromal infiltrate around the original $1 \times 1 \mathrm{~mm}$ ulcer and two small satellite lesions in the mid-periphery. Symptoms persisted, and the presumptively contaminated contact lens was brought to the

\section{KARGER}

David B. Warner, MD

Department of Ophthalmology, University of Arkansas for Medical Sciences

4301 W. Markham Street, Slot 523

Little Rock, AR 72205 (USA)

E-Mail dbwarner@uams.edu 
Warner et al.: Rhizopus Keratitis Associated with Poor Contact Lens Hygiene

clinic and cultured for microorganisms. The laboratory identified a rapidly growing mold, of the order Mucorales (class Zygomycetes), which was further characterized as Rhizopus spp. (fig. 1). Hourly amphotericin B $0.15 \%$ drops were initiated with rapid initial clinical improvement of the corneal infiltrate over the next 2 days.

The patient was lost to follow-up and returned approximately 1 month later. The stromal infiltrate had partially resolved with scarring at the periphery and now contained fine intrastromal crystals (fig. 2). Her vision remained stable, and amphotericin drops were decreased to every $2 \mathrm{~h}$. For the next 2.5 months, she failed to return for subsequent appointments due to financial constraints. At her next visit, the infiltrate had completely scarred, and the best spectacle corrected visual acuity was 20/60. She was referred for contact lens fitting and achieved $20 / 25$ vision with a scleral lens.

\section{Discussion}

Molds, or filamentous fungi, can be divided into septate and nonseptate forms. Septate filamentous fungi, specifically Fusarium and Aspergillus, are the most frequent causes of mycotic keratitis, as high as 16-40 and 17-59\%, respectively [1]. For treatment of filamentous fungal keratitis, natamycin provides superior clinical outcomes compared to voriconazole, including best corrected visual acuity, lesser chance of corneal perforation, and reduced need for penetrating keratoplasty [2]. Within the order Mucorales, there are four notable genera pathogenic to humans. These include Rhizopus, Mucor, Rhizomucor, and Absidia, all of which are found widely in the environment. These molds typically have broad, nonseptate hyphae and grow rapidly in laboratory conditions. Clinically, they are not commonly encountered in ophthalmologic practice; however, they can present as rhinocerebral and extraocular infections in the immunocompromised, and much less commonly as ocular infections (e.g. keratitis) [3].

We are aware of only two reported cases of Rhizopus keratitis. Schwartz et al. [4] reported the first case in 1978, a 24-year-old male presenting with a perforating injury from a soil-contaminated screw driver. The open globe, initially repaired and treated with steroids and broad-spectrum intravenous antibiotics, presented 1.5 months later as a fulminant keratitis and anterior uveitis. The patient required a penetrating keratoplasty after failing treatment with natamycin drops and oral flucytosine. Intravenous amphotericin B was initiated and provided eventual clinical improvement. In 2013, Azari et al. [5] reported a case of Rhizopus keratitis in a 48-year-old male presenting after trauma from a metal wire. The patient was treated successfully with oral and topical voriconazole.

To our knowledge, our case is the first patient reported to have Rhizopus keratitis associated with contact lens wear. Although Gram stain of the corneal scraping and culture were negative for fungi, these tests are the least sensitive for fungal keratitis [6], and clinical presentation coupled with contact lens culture and response to antifungal therapy confirm the diagnosis. While Rhizopus is known to cause rapid and severe destruction of the extraocular and rhinocerebral soft tissues, our observation is that it behaves less aggressively in the cornea, perhaps due to the avascularity of this tissue. In our patient, the mold was highly sensitive to topical amphotericin when administered following a relatively prompt diagnosis.

We recognize that the culture came from a contact lens rather than a corneal scraping, and that we cannot be certain that the organism from the lens we cultured corresponds to the corneal infection. However, it is compelling that the patient's rapid clinical response strongly coincided with the appropriate treatment based on the contact lens culture. 
Warner et al.: Rhizopus Keratitis Associated with Poor Contact Lens Hygiene

This case underscores the importance of considering fungi in the differential diagnosis for patients with contact lens-related infiltrates who demonstrate an insufficient clinical response to empiric antibiotic therapy. We recommend obtaining cultures of both the contact lens and cornea early in the course of treatment to avoid delay in proper treatment. Empiric antifungal treatment may also be indicated depending on presentation, individual laboratory capabilities, and laboratory turnaround times.

\section{Statement of Ethics}

The authors have no ethical conflicts to disclose.

\section{Disclosure Statement}

The authors have no conflicts of interest.

\section{References}

1 Punia RS, Kundu R, Chander J, Arya SK, Handa U, Mohan H: Spectrum of fungal keratitis: clinicopathologic study of 44 cases. Int J Ophthalmol 2014;7:114-117.

-2 Prajna NV, Krishnan T, Mascarenhas J, Rajaraman R, Prajna L, Srinivasan M, Raghavan A, Oldenburg CE, Ray KJ, Zegans ME, McLeod SD, Porco TC, Acharya NR, Lietman TM; Mycotic Ulcer Treatment Trial Group: The mycotic ulcer treatment trial: a randomized trial comparing natamycin vs voriconazole. JAMA Ophthalmol 2013;131:422-429.

3 Thomas PA, Geraldine P: Oculomycosis; in Collier L, Balows A, Sussman M (eds): Topley and Wilson's Microbiology and Microbial Infections, ed 10. London, Hodder Arnold, 2005, chap 16.

4 Schwartz LK, Loignon LM, Webster RG Jr: Posttraumatic phycomycosis of the anterior segment. Arch Ophthalmol 1978;96:860-863.

5 Azari AA, Nehls S, Ghoghawala SY, Lee V, Kanavi MR, Potter HD: Rhizopus keratitis following corneal trauma. JAMA Ophthalmol 2013;131:776.

-6 Badiee P, et al: Comparative study of Gram stain, potassium hydroxide smear, culture and nested PCR in the diagnosis of fungal keratitis. Ophthalmic Res 2010;44:251-256. 


\section{Case Reports in \\ Ophthalmology}

\begin{tabular}{l|l}
\hline Case Rep Ophthalmol 2016;7:21-24 \\
\hline DOI: 10.1159/000443325 & $\begin{array}{l}\text { @ 2016 The Author(s). Published by S. Karger AG, Basel } \\
\text { www.karger.com/cop }\end{array}$ \\
\hline
\end{tabular}

Warner et al.: Rhizopus Keratitis Associated with Poor Contact Lens Hygiene

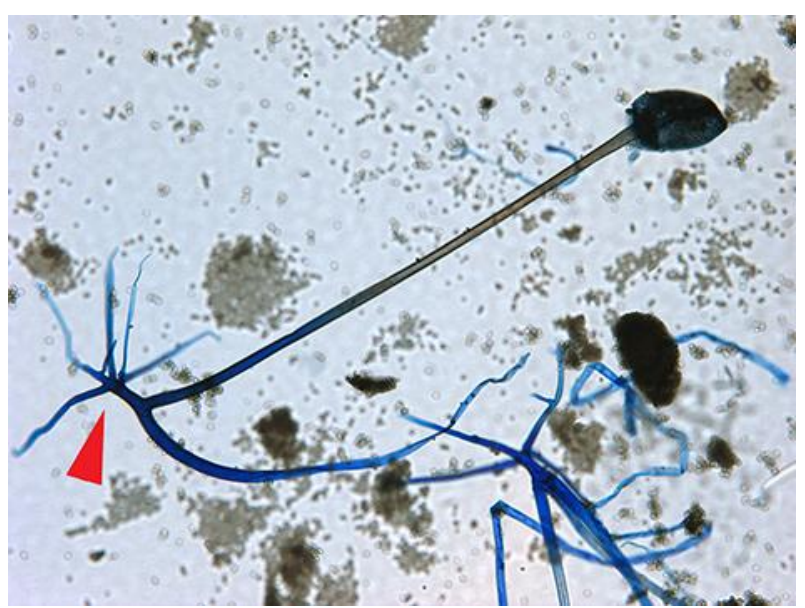

Fig. 1. Contact lens culture. Lactophenol cotton blue slide preparation, $\times 1,000$. Rhizopus spp. demonstrating rhizoids (arrow head) emerging characteristically at the base of a sporangiophore.

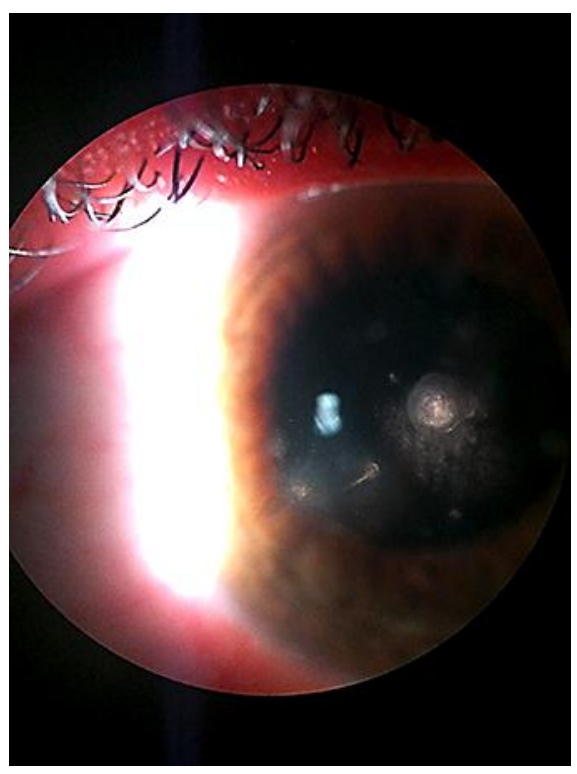

Fig. 2. Slit-lamp photograph of resolving corneal infiltrate and satellite lesions after 1 month of treatment, using the Apple iPhone 4. 